\title{
The fountain of youth: Influence of human leukocyte antigen compatibility in adult and pediatric lung transplantation
}

\author{
David D. Odell, MD, MMSc
}

\footnotetext{
From Division of Thoracic Surgery, Institute for Public Health and Medicine, Feinberg School of Medicine, Northwestern University, Chicago, Ill.

Disclosures: Author has nothing to disclose with regard to commercial support.

Received for publication Aug 25, 2015; accepted for publication Aug 25, 2015; available ahead of print Sept 26, 2015.

Address for reprints: David D. Odell, MD, MMSc, Division of Thoracic Surgery, Surgical Outcomes and Quality Improvement Center, Northwestern University, Feinberg School of Medicine, 676 N St. Clair St, Suite 650, Chicago, IL 60611 (E-mail: dodell@nm.org).

J Thorac Cardiovasc Surg 2016;151:558-9

$0022-5223 / \$ 36.00$

Copyright $(2016$ Published by Elsevier Inc. on behalf of The American Association for Thoracic Surgery http://dx.doi.org/10.1016/j.jtcvs.2015.08.094
}

The relationship between the cellular immune system and lung allograft rejection is well described. However, our understanding of the effect of the humoral immune system after lung transplantation remains relatively poor. Although human leukocyte antigen (HLA) compatibility has been shown to play an important role in renal transplantation ${ }^{1}$ and cardiac transplantation ${ }^{2}$ for several years, the existence of a similar, antibody-mediated rejection phenomenon in the lung was questioned until recently. The process is characterized by recognition of donor HLA antigens by the recipient humoral immune system, resulting in activation of the complement cascade and ultimately endothelial injury and tissue inflammation. ${ }^{3}$ The development of donor-specific anti-HLA antibodies has now been associated with both the development of bronchiolitis obliterans syndrome and with death following lung transplantation. ${ }^{4}$ Whether the development of donor-specific anti-HLA antibodies can be predicted by the extent of HLA mismatch between donor and recipient remains unclear, although many groups have shown worse outcomes with increasing HLA mismatch.

In this issue of the Journal, Hayes and colleagues ${ }^{5}$ present an intriguing analysis of the United Network for Organ Sharing Standard Transplant Analysis and Research database examining the influence of HLA mismatch following lung transplantation for cystic fibrosis (CF). The authors previously used this dataset to examine the influence of HLA matching in a mixed lung transplant cohort demonstrating that mismatch of $\geq 3$ loci or either the HLA-A or HLA-DR locus was associated with an increased risk of death after lung transplantation. ${ }^{6}$ The present study ${ }^{5}$ was conceived to more deeply investigate the observation that there appears to be an age-dependent component to outcome following transplantation in patients with CF. The authors examined outcomes for 3149 adult (aged $\geq 18$ years) and 489 pediatric (aged $<18$ years) patients with $\mathrm{CF}$, assessing a primary survival outcome as a function of the element of bias.

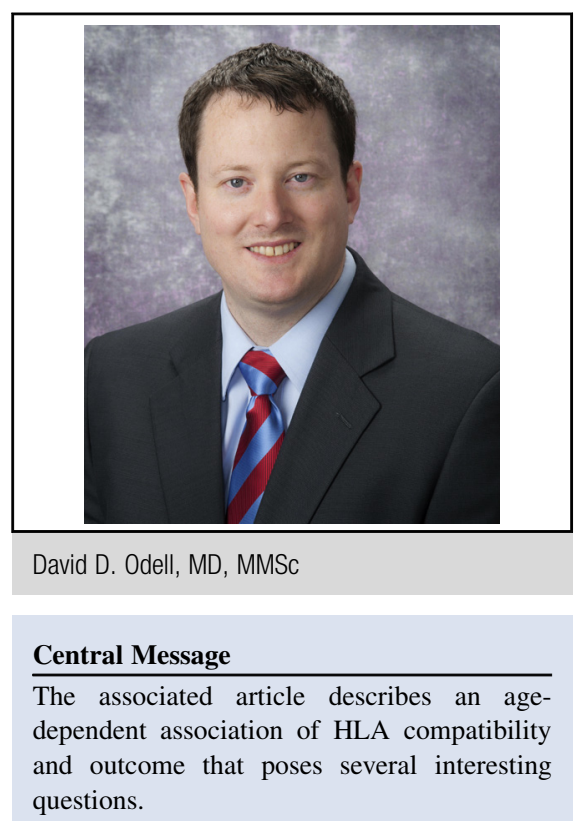

See Article page 549.

See Editorial page 315 .

extent of HLA mismatch between donor and recipient. The principle finding of the study is that an increase in donor-recipient HLA mismatch was protective in the pediatric patients in both univariate (hazard ratio, 0.87; $P=.0032$ ) and multivariate (hazard ratio, 0.85; $P=.044)$ analyses with the protective effect dissipating by roughly age 10 years. The concept of relating donor age to tolerance of HLA mismatch has not previously been studied and the observed protective effect of mismatching in the pediatric population in this study is in contrast to the results of prior work in adult populations by these authors as well as other groups. However, the pediatric population in this study represents a small subset of the overall CF cohort. Further, because the protective effect of mismatching is only seen in those patients younger than age 10 years, readers should take caution that the small sample may potentially introduce an

Although the findings are provocative, the study opens the door to several interesting questions rather than yielding a complete understanding of the role of HLA compatibility in patients with CF. If the immune system is indeed more tolerant of antigenic variability in young 
people, this finding might provide an opportunity to examine alternative strategies for posttransplant immunomodulation. The concept that increased HLA mismatching, and hence antigenic exposure, may be in some way protective in specific populations represents a paradigm shift in lung transplantation. It is wise to be cautious in considering these findings, yet the implications are significant in terms of the potential to direct future investigations in transplant immunobiology. An improved understanding of the relationship of HLA compatibility between donor and recipient and outcomes has wide-ranging implications for donor selection, organ availability, and potentially for alterations in our approach to immunosuppression following transplantation.

\section{References}

1. Opelz G, Mytilineos J, Wujciak T, Schwarz V, Back D. Current status of HLA matching in renal transplantation. The Collaborative Transplant Study. Clin Investig. 1992;70:767-72

2. Opelz G, Wujciak T. The influence of HLA compatibility on graft survival after heart transplantation. The Collaborative Transplant Study. N Engl J Med. 1994; 330:816-9.

3. McManigle W, Pavlisko EN, Martinu T. Acute cellular and antibody-mediated allograft rejection. Semin Respir Crit Care Med. 2013;34:320-35.

4. Morrell MR, Pilewski JM, Gries CJ, Pipeling MR, Crespo MM, Ensor CR, et al De novo donor-specific HLA antibodies are associated with early and highgrade bronchiolitis obliterans syndrome and death after lung transplantation. $J$ Heart Lung Transplant. 2014;33:1288-94.

5. Hayes D Jr, Auletta JJ, Whitson BA, Black SM, Krikby S, Tobias JD Mansour HM. HLA mismatching and survival in adult and pediatric patients with cystic fibrosis. J Thorac Cardiovasc Surg. 2016;151:549-57.

6. Hayes D Jr, Whitson BA, Ghadiali SN, Tobias D, Mansour HM, Black SM. Influence of HLA mismatching on survival in lung transplantation. Lung. July 29, 2015 [Epub ahead of print]. 